\title{
Neutron-fed afterglows of gamma-ray bursts
}

\author{
Andrei M. Beloborodov ${ }^{1,2,3}$
}

\begin{abstract}
The baryonic material ejected in a gamma-ray burst (GRB) is partially made of free neutrons. At late stages of the explosion, the neutrons fully decouple from the ion ejecta and coast freely. Their presence profoundly changes the mechanism of the GRB afterglow. The neutrons lead the decelerating blast wave and gradually decay, leaving behind a trail of the decay products mixed with the ambient medium. The kinetic energy of the decay products far exceeds the medium rest energy, and the trail has a Lorentz factor $\gamma \gg 1$ at radii $R \lesssim R_{\text {trail }} \approx 10 R_{\beta} \approx 10^{17} \mathrm{~cm}$, where $R_{\beta}$ is the mean radius of neutron decay. The ion ejecta decelerate behind as they sweep up the neutron trail and drive a shock wave into it. The afterglow is emitted by the preshock and postshock parts of the neutron trail. The neutron-fed afterglow can naturally rebrighten at $R \approx R_{\text {trail }}$. Absence of any neutron signatures would point to an extremely low baryon loading of the GRB ejecta and a strong dominance of the Poynting flux.
\end{abstract}

Subject headings: Cosmology: miscellaneous — gamma-rays: bursts — radiation mechanisms: nonthermal — shock waves

\section{Introduction}

The central engines of gamma-ray bursts (GRBs) produce neutrons that can affect the observed explosion (Derishev, Kocharovsky, \& Kocharovsky 1999a,b; Bahcall \& Mészáros 2000; Mészáros \& Rees 2000; Fuller, Pruet, \& Abazajian 2000; Pruet \& Dalal 2002). In an accompanying paper (Beloborodov 2002, Paper 1) we study in detail the nuclear composition of the GRB fireballs and show that the presence of neutrons among the ejected baryons is practically inevitable. One implication is an observable multi-GeV neutrino emission from inelastic neutron-ion collisions in the ejecta. Here, we focus on a different aspect. We show that the neutrons have a dramatic impact on the explosion dynamics at radii as large as $10^{17} \mathrm{~cm}$ and propose a novel mechanism for the GRB afterglow emission.

Let us remind what happens in a standard relativistic explosion without neutrons (see Mészáros 2002 for a review). The ejecta with mass $M_{\mathrm{ej}}$ and Lorentz factor $\Gamma_{\mathrm{ej}}$ sweep up the ambient medium

\footnotetext{
${ }^{1}$ Canadian Institute for Theoretical Astrophysics, 60 St. George Street, Toronto, ON M5S 3H8, Canada

${ }^{2}$ Physics Department, Columbia University, 538 West 120th Street New York, NY 10027

${ }^{3}$ Astro-Space Center of Lebedev Physical Institute, Profsojuznaja 84/32, Moscow 117810, Russia
} 
and gradually dissipate their kinetic energy. The dissipation rate peaks at a characteristic "deceleration" radius $R_{\text {dec }}$ where half of the initial energy is dissipated. This radius corresponds to the swept-up mass $m_{\mathrm{dec}}=M_{\mathrm{ej}} / \Gamma_{\mathrm{ej}}$. Further dynamics is described by the self-similar blast wave model of Blandford \& McKee (1976). How does this picture change in the presence of neutrons?

The neutrons develop Lorentz factor $\Gamma_{n}=10^{2}-10^{3}$ at the very beginning of the explosion when the fireball is accelerated by radiation pressure (Derishev et al. 1999b). They are collisionally coupled to the ions in the early dense fireball, and decouple close to the end of the acceleration stage. Then the neutrons coast and gradually decay with the mean lifetime $\tau_{\beta} \approx 900 \mathrm{~s}$ and the mean decay radius $R_{\beta}=c \tau_{\beta} \Gamma_{n}$,

$$
R_{\beta}=0.9 \times 10^{16}\left(\frac{\Gamma_{n}}{300}\right) \mathrm{cm} .
$$

Surprisingly, the new "neutron-fed" mechanism of the afterglow operates at radii up to $10 R_{\beta} \approx$ $10^{17} \mathrm{~cm}$, which likely covers the major stage of the afterglow. An outline of the mechanism is as follows. At radii under consideration, $R>10^{15} \mathrm{~cm}$, the $\mathrm{n}$ - and i- components of the ejecta can be viewed as two decoupled thin shells (the ejecta thickness is $\Delta \ll R$ ). The $n$-shell coasts with a constant $\Gamma_{n}$ and leads the decelerating blast wave. It gradually decays and leaves behind a trail of decay products mixed with the ambient medium. The trail is formed relativistically hot and moving with a high Lorentz factor $1 \ll \gamma<\Gamma_{n}$. The decelerating i-shell follows with a Lorentz factor $\Gamma$ $\left(\gamma<\Gamma<\Gamma_{n}\right)$ and drives a shock wave into the trail.

\section{Neutron front and its trail}

As the ion component of the ejecta decelerates, it falls behind and separates from the neutrons. Thus, the ejected neutrons and ions form two distinct shells, which we call the $\mathrm{n}$ - and $\mathrm{i}$ - shells (the n-shell will also be called "neutron front"). The i-shell lags behind by a distance $l$,

$$
\frac{l}{R} \approx \beta_{n}-\beta_{i} \approx \frac{1}{2 \Gamma^{2}}-\frac{1}{2 \Gamma_{n}^{2}}
$$

where $\beta_{i}$ and $\beta_{n}$ are the shell velocities in units of $c$, and $\Gamma$ and $\Gamma_{n}$ are their Lorentz factors. The shells are completely separated when $l$ exceeds their thickness $\Delta$. For simplicity, we hereafter consider the stage with $l>\Delta$. It covers the whole explosion if $\Delta \rightarrow 0$.

The mass of the $\mathrm{n}$-shell gradually decreases because of the $\beta$-decay,

$$
M_{n}(R)=M_{n}^{0} \exp \left(-\frac{R}{R_{\beta}}\right)
$$

The n-shell energy, $E_{n}=\Gamma_{n} M_{n} c^{2}$, is huge compared to the ambient rest mass, $m c^{2}$. For example, at $R=R_{\mathrm{dec}}, E_{n} / m_{\mathrm{dec}}=X_{n} \Gamma_{n}^{2} \exp \left(-R_{\mathrm{dec}} / R_{\beta}\right)$, where the $X_{n}=M_{n}^{0} / M_{\mathrm{ej}}$. The decay products $p$ and $e^{-}$share immediately their huge momentum with the ambient particles due to the two-stream 
instability (the timescale of the plasma processes is set by the ion plasma frequency $\omega_{i}$, and it is the shortest timescale in the problem). Thus, the n-shell leaves behind a trail with a relativistic bulk velocity $\beta<\beta_{n}$, which we calculate now.

Let $\mathrm{d} m=(\mathrm{d} m / \mathrm{d} R) \mathrm{d} R$ be the ambient mass overtaken by the $\mathrm{n}$-shell as it passes $\mathrm{d} R$ and $\mathrm{d} M_{n}=\left(M_{n} / R_{\beta}\right) \mathrm{d} R$ be the mass of decayed neutrons. The $\mathrm{d} m$ and $\mathrm{d} M_{n}$ share momentum and form a trail element with proper mass $\mathrm{d} m_{*}=\mathrm{d} M_{n}+\mathrm{d} m+\mathrm{d} m_{\text {heat }}$, which includes heat dissipated in the inelastic $\mathrm{d} m-\mathrm{d} M_{n}$ collision. The laws of energy and momentum conservation read

$$
\Gamma_{n} \mathrm{~d} M_{n}+\mathrm{d} m=\gamma \mathrm{d} m_{*}, \quad \beta_{n} \Gamma_{n} \mathrm{~d} M_{n}=\beta \gamma \mathrm{d} m_{*},
$$

where $\gamma=\left(1-\beta^{2}\right)^{-1 / 2}$ is the trail Lorentz factor. Let us denote

$$
\zeta(R)=\frac{\mathrm{d} M_{n}}{\mathrm{~d} m}=\frac{M_{n}}{R_{\beta}}\left(\frac{\mathrm{d} m}{\mathrm{~d} R}\right)^{-1} .
$$

From equations (4) we find

$$
\beta(R)=\frac{\beta_{n}}{1+\left(\Gamma_{n} \zeta\right)^{-1}}, \quad \gamma(R)=\frac{\Gamma_{n} \zeta+1}{\left(\zeta^{2}+2 \Gamma_{n} \zeta+1\right)^{1 / 2}} .
$$

It gives $\gamma \gg 1$ until essentially all neutrons have decayed. To illustrate this important point let us specialize to a power-law density profile of the ambient medium. Then

$$
m(R)=m_{\beta}\left(\frac{R}{R_{\beta}}\right)^{k}, \quad \zeta(R)=\frac{M_{n}}{k m_{\beta}}\left(\frac{R}{R_{\beta}}\right)^{1-k} .
$$

$\zeta$ evolves from $\zeta \sim M_{n}^{0} / m_{\beta} \gg 1$ at $R<R_{\beta}$ to $\zeta \ll 1$ at $R \gg R_{\beta}$ as $M_{n}$ decays exponentially. There exists a characteristic radius $R_{\text {trail }}$ where $\gamma$ decreases to unity, and it is defined by condition $\zeta=\Gamma_{n}^{-1}$ (eq. 6). This requires about 10 e-foldings of the decay (for typical $m_{\beta} \sim m_{\mathrm{dec}} \sim 10^{-5} M_{n}^{0}$ ). Thus,

$$
R_{\text {trail }} \approx 10 R_{\beta} \approx 10^{17}\left(\frac{\Gamma_{n}}{300}\right) \mathrm{cm}
$$

$R_{\text {trail }}$ very weakly depends on the ambient density. It also weakly depends on the initial neutron fraction $X_{n}$ as long as $X_{n} \gg \Gamma_{n}^{-2}$. $R_{\text {trail }}$ has practically equal values for $X_{n}=0.9$ and $X_{n}=0.01$.

We now calculate the trail density. The n-shell is thin and the ambient particles cross it almost instantaneously (on timescale $\gamma^{2} \Delta / c \ll R / c$ ). Measured in the n-shell frame, the flux of ambient particles is

$$
\beta_{n} \Gamma_{n} n_{0}=\left(\beta_{n}-\beta\right) \Gamma_{n} \gamma n_{\mathrm{amb}}
$$

Here $n_{0}$ and $n_{\mathrm{amb}}$ are the proper densities of the ambient particles ahead and behind the n-shell, respectively. The total density of the trail, $n=(1+\zeta) n_{\mathrm{amb}}$, includes the neutron decay products. Using equation (6), we find

$$
\frac{n}{n_{0}}=\frac{\beta_{n}(1+\zeta)}{\gamma\left(\beta_{n}-\beta\right)}=(1+\zeta)\left(\zeta^{2}+2 \Gamma_{n} \zeta+1\right)^{1 / 2}
$$


When $\zeta<1$, the density of the decay products is small, and the density enhancement, $n \approx n_{\mathrm{amb}}$, is solely due to compression of the ambient medium accelerated in the neutron front.

The energy dissipated in the neutron front is given by

$$
\mathrm{d} E_{\text {n.f. }}=\gamma\left(\mathrm{d} m_{*}-\mathrm{d} m-\mathrm{d} M_{n}\right) c^{2}=\left(\Gamma_{n}-\gamma\right) \mathrm{d} M_{n} c^{2}-(\gamma-1) \mathrm{d} m c^{2} .
$$

After simple algebra (using eqs. 4), we find

$$
\frac{\mathrm{d} E_{\text {n.f. }}}{\mathrm{d} R}=\left[\frac{\Gamma_{n} \beta_{n}-\gamma \beta}{\Gamma_{n} \gamma\left(\beta_{n}-\beta\right)}-1\right] \gamma \frac{\mathrm{d} m}{\mathrm{~d} R} c^{2} .
$$

It simplifies to $\mathrm{d} E_{\text {n.f. }}=2 \gamma^{2} \mathrm{~d} m c^{2}$ for the interesting regime $1 \ll \gamma \ll \Gamma_{n}\left(\Gamma_{n}^{-1}<\zeta<\Gamma_{n}\right)$. The trail is formed very hot. This can be seen when comparing inertial mass $\mathrm{d} m_{*}$ (that includes heat) with rest mass $\mathrm{d} M_{b}=\mathrm{d} m+\mathrm{d} M_{n}=(1+\zeta) \mathrm{d} m$, which gives the dimensionless relativistic enthalpy of the trail,

$$
\mu=\frac{\mathrm{d} m_{*}}{\mathrm{~d} M_{b}}=\frac{\left(\zeta^{2}+2 \Gamma_{n} \zeta+1\right)^{1 / 2}}{1+\zeta} .
$$

For $\Gamma_{n}^{-1}<\zeta<\Gamma_{n}$, we find $\mu \gg 1$, i.e. the internal energy of the trail far exceeds its rest energy. The trail parameters are summarized in Table 1.

It is instructive to view the dissipation process in the rest frame of the trail. Here, the elements $\mathrm{d} m$ and $\mathrm{d} M_{n}$ have initial Lorentz factors $\gamma$ and $\tilde{\gamma}=\Gamma_{n} \gamma\left(1-\beta \beta_{n}\right)$, share their opposite momenta, and come at rest. This is achieved via the plasma instability that isotropizes the particle momentum distribution. It may result in two isotropic components: the ambient ions with mean Lorentz factor $\gamma$ and the $\beta$-decay protons with mean Lorentz factor $\tilde{\gamma}$. Thus, the trail may be composed of two ion populations with different temperatures. Both populations are relativistically hot, $\gamma \gg 1$ and $\tilde{\gamma} \gg 1$, as long as $\Gamma_{n}^{-1}<\zeta<\Gamma_{n}$. They have equal energies $\tilde{\gamma} \mathrm{d} M_{n}=\gamma \mathrm{d} m$ (which correspond to equal relativistic bulk momenta of $\mathrm{d} M_{n}$ and $\mathrm{d} m$ before they come at rest in the trail frame). The ratio of their temperatures is the reciprocal of their density ratio.

A detailed model of the dissipation in the neutron front is an interesting plasma physics problem, which we defer to a future work. We emphasize here that it is different from dissipation

Table 1: Trail parameters

\begin{tabular}{ccccc}
\hline \hline & $\zeta>\Gamma_{n}$ & $1<\zeta<\Gamma_{n}$ & $\Gamma_{n}^{-1}<\zeta<1$ & $\zeta<\Gamma_{n}^{-1}$ \\
\hline$\gamma$ & $\Gamma_{n}$ & $\left(\Gamma_{n} \zeta / 2\right)^{1 / 2}$ & $\left(\Gamma_{n} \zeta / 2\right)^{1 / 2}$ & 1 \\
$n / n_{0}$ & $\zeta^{2}$ & $2 \gamma \zeta$ & $2 \gamma$ & 1 \\
$\mu$ & 1 & $\Gamma_{n} / \gamma \approx 2 \tilde{\gamma}$ & $2 \gamma$ & 1 \\
\hline
\end{tabular}


in collisionless shocks. The thickness of a standard shock, $\delta \sim 10 c / \omega_{i}$, is set by the instability timescale, and it is a discontinuity in the hydrodynamical sense. By contrast, the n-shell has thickness $\Delta \sim 10^{11}-10^{12} \mathrm{~cm}$, which is 5-7 orders of magnitude larger than $\delta$. The neutrons decay and cause dissipation everywhere in the n-shell. The medium velocity grows smoothly from 0 at the leading edge to $\beta$ at the trailing edge of the $\mathrm{n}$-shell. The dissipation here can be called "volume dissipation".

\section{Shock wave}

The i-shell follows the n-shell and collects its trail. As a result (1) the Lorentz factor of the i-shell, $\Gamma$, decreases, and (2) a shock wave propagates into the trail material. The shock front is between the i- and n- shells; it has a Lorentz factor $\Gamma \lesssim \Gamma_{\text {sh }}<\Gamma_{n}$ and cannot catch up with the neutron front (unless $n_{0}[R]$ falls off steeper than $R^{-3}$ ). Given the shell separation $l$ (eq. 2) we find the time it takes the i-shell to pick up the trail material,

$$
t_{i}=\frac{l}{c\left(\beta_{i}-\beta\right)} \approx \frac{\gamma^{2}}{\left(\Gamma^{2}-\gamma^{2}\right)} \frac{R}{c}
$$

It is much shorter than $R / c$ as long as $\Gamma \gg \gamma$. Hereafter we use the approximation $t_{i}<R / c$ and assume that the trail is picked up before it could expand and change its density or velocity. We allow, however, for rapid radiative losses because the trail may cool on a timescale $t_{\text {cool }} \ll R / c$ and possibly $t_{\text {cool }}<t_{i}$. Below we considers two extreme cases: $t_{\text {cool }} \gg t_{i}$ ("adiabatic" trail) and $t_{\text {cool }} \ll t_{i}$ ("radiative" trail with $\mu=1$ ).

In the radiative regime, the energy dissipated by the shock is

$$
\frac{\mathrm{d} E_{\mathrm{sh}}}{\mathrm{d} R}=\Gamma\left(\Gamma_{\text {rel }}-1\right)(1+\zeta) \frac{\mathrm{d} m}{\mathrm{~d} R} c^{2}
$$

where $\Gamma_{\text {rel }}=\Gamma \gamma\left(1-\beta_{i} \beta\right)$ is the trail Lorentz factor with respect to the i-shell. For $\Gamma_{n}^{-1} \ll \zeta \ll \Gamma_{n}$ one can use the approximate expressions $\zeta=2 \gamma^{2} / \Gamma_{n}$ and $\Gamma_{\text {rel }}=(1 / 2)(\gamma / \Gamma+\Gamma / \gamma)$ to get

$$
\frac{\mathrm{d} E_{\mathrm{sh}}}{\mathrm{d} R}=\left(\frac{1}{2 \gamma}+\frac{\gamma}{\Gamma_{n}}\right)(\Gamma-\gamma)^{2} \frac{\mathrm{d} m}{\mathrm{~d} R} c^{2} .
$$

Note that the dissipation is smaller than it would be in the absence of the neutron front, $\mathrm{d} E_{\mathrm{sh}}=$ $\Gamma(\Gamma-1) \mathrm{d} m c^{2}$. Hence, in the radiative regime, the neutrons delay the i-shell deceleration.

In the adiabatic regime, the preshock material has enthalpy $\mu$ given by equation (13). The postshock heat, measured in the lab frame, is $\mathrm{d} E_{\text {heat }}=\Gamma\left(\Gamma_{\text {rel }} \mu-1\right)(1+\zeta) \mathrm{d} m$. It includes heat deposited by the neutron front (eq. 11), which should be subtracted to get the energy dissipated in the shock itself. Hence,

$$
\frac{\mathrm{d} E_{\mathrm{sh}}}{\mathrm{d} R}=\left[\Gamma\left(\Gamma_{\text {rel }} \mu-1\right)-\gamma(\mu-1)\right](1+\zeta) \frac{\mathrm{d} m}{\mathrm{~d} R} c^{2} .
$$


For $1 \ll \gamma<\Gamma_{n}$ we get $\mathrm{d} E_{\text {n.f. }}=2 \gamma^{2} \mathrm{~d} m c^{2}, \mathrm{~d} E_{\text {sh }}=\left(\Gamma^{2}-\gamma^{2}\right) \mathrm{d} m c^{2}$, and the total dissipated energy $\mathrm{d} E_{\text {heat }}=\left(\Gamma^{2}+\gamma^{2}\right) \mathrm{d} m c^{2}$. As long as $\Gamma \gg \gamma$, the bulk of energy is dissipated in the shock rather than in the neutron front. Moreover, the dissipated energy is the same as in the absence of the neutron front, and hence the i-shell deceleration is the same.

In both radiative and adiabatic regimes, the shock dissipation is suppressed when the blast wave decelerates to $\Gamma \sim \gamma$. At $\Gamma=\gamma$ the shock would disappear completely. Thus, $\Gamma$ is bound from below by $\gamma$.

\section{Numerical examples}

Let us consider the simple radiative regime. The mass of the i-shell $M(R)=M_{\mathrm{ej}}+m-M_{n}$ is a known function of radius. It grows as the i-shell picks up the trail material. Mass gain $\mathrm{d} M$ causes deceleration $\mathrm{d} \Gamma$ that is found from the energy and momentum conservation: $\mathrm{d}(\Gamma M)=$ $\gamma \mathrm{d} M-\mathrm{d} E_{\text {rad }} / c^{2}$ and $\mathrm{d}\left(\beta_{i} \Gamma M\right)=\beta \gamma \mathrm{d} M-\left(\mathrm{d} E_{\text {rad }} / c^{2}\right) \beta_{i}$, where $\mathrm{d} E_{\text {rad }}=\mathrm{d} E_{\text {sh }}$ is the radiated energy. Excluding $\mathrm{d} E_{\text {rad }}$, we get the dynamic equation for the i-shell,

$$
M \frac{\mathrm{d} \Gamma}{\mathrm{d} R}=-\Gamma^{2} \gamma \beta_{i}\left(\beta_{i}-\beta\right)(1+\zeta) \frac{\mathrm{d} m}{\mathrm{~d} R} .
$$

Using equations (6) and (7) we can solve numerically equation (18) for $\Gamma(R)$ with an initial condition $\Gamma_{\text {ej }}$. The results depend on the type of the ambient medium. Figure 1 shows two examples: a standard ISM and a wind from a Wolf-Rayet progenitor.

The explosion has two separate emission regions: behind the neutron front and behind the shock front. They have different luminosities and spectra. In the radiative model, the luminosities equal the corresponding dissipation rates $\left(c \mathrm{~d} E_{\text {n.f. }} / \mathrm{d} R\right)$ and $\left(c \mathrm{~d} E_{\mathrm{sh}} / \mathrm{d} R\right)$ (Fig. 1). The neutron front dissipation peaks at $R_{\beta}$. The shock dissipation can have two peaks (if $m_{\beta} \gg M_{\mathrm{ej}} / \Gamma_{\mathrm{ej}}$, as in the wind example in Fig. 1). The first peak marks the beginning of the i-shell deceleration, and it is followed by a minimum when $\Gamma$ approaches $\gamma$. At $R>2 R_{\beta}, \gamma$ falls down steeply, and the shock becomes strong again (high $\Gamma_{\text {rel }}$ ). The second peak is reached at $R \approx R_{\text {trail }}$ where $\gamma \sim 1$.

\section{Discussion}

The dynamics of a neutron-fed explosion is a relatively clean physical problem. The existence of neutrons in GRBs and their mean lifetime of $15 \mathrm{~min}$ is all we needed to construct the model. We focused here on the simplest case where all neutrons have equal Lorentz factor $\Gamma_{n}$, and data fits may require a multi-shell picture with variable $\Gamma_{n}$. It would confirm the variability of the central

engines in GRBs that is thought to cause internal shocks in the ejecta and produce the prompt $\gamma$-ray emission (Mészáros 2002). The variability would affect the presented model in case of high variations $\Delta \Gamma_{n}>\Gamma_{n}$. Another complication is related to the likely beaming of the ejecta. At late stages, the ion ejecta spread laterally, while the neutron beaming remains constant. 


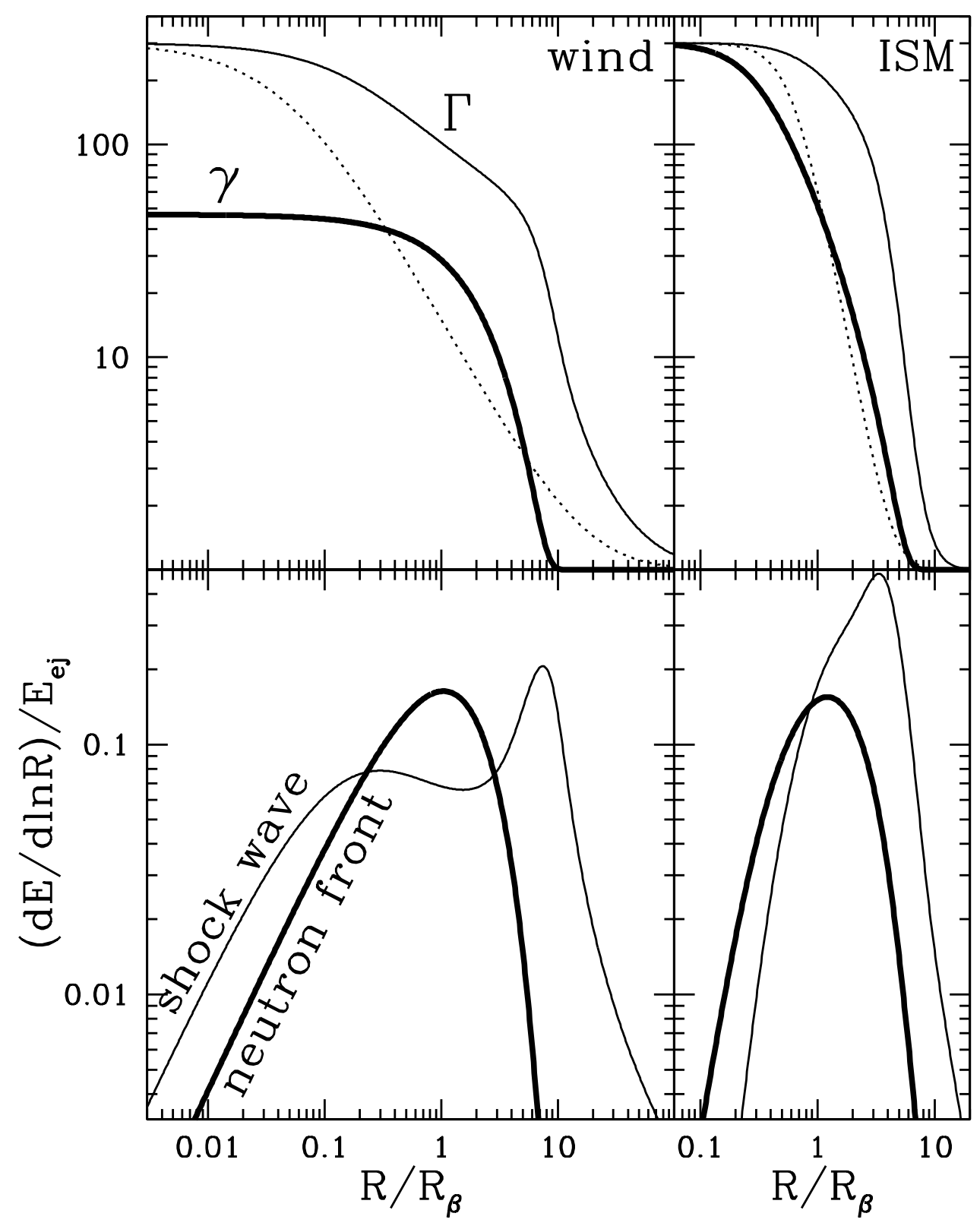

Fig. 1. - Radiative model with $X_{n}=0.5$ and $\Gamma_{n}=\Gamma_{\mathrm{ej}}=300$. Left panels show the results for $m(R)$ that is typical for a Wolf-Rayet progenitor $\left(k=1\right.$ and $\left.m_{\beta}=10 M_{\mathrm{ej}} / \Gamma_{\mathrm{ej}}\right)$. Right panels show the ISM case with $k=3$ and $m_{\beta}=M_{\mathrm{ej}} / \Gamma_{\mathrm{ej}}$. Top: Trail Lorentz factor $\gamma$ and the i-shell Lorentz factor $\Gamma$. The dotted curve shows the i-shell deceleration $\Gamma(R)$ that would take place without neutrons. Bottom: Radial distribution of the dissipation rate in the neutron front and the shock. $E_{\mathrm{ej}}=\Gamma_{\mathrm{ej}} M_{\mathrm{ej}} c^{2}$ is the initial total energy of the ejecta. Radius $R$ is measured in units of the mean decay radius $R_{\beta}$ (eq. 1 ). 
In contrast to dynamics, the emission of neutron-fed blast waves is really complicated. Like a standard blast wave, the expected radiation is difficult to derive from first principles. The afterglow radiation is believed to be synchrotron, and it depends on poorly understood generation of magnetic field and electron acceleration. The standard model without neutrons relies on the field generation in the shock by the two-stream instability (Sagdeev 1966, Medvedev \& Loeb 1999, Gruzinov 2001). The shock front is, however, extremely thin $\left(\delta \approx 10 c / \omega_{i}\right)$, and the postshock field decays quickly. The model needs a significant remnant field to survive in an extended layer behind the shock, which is uncertain. This problem can be alleviated in a neutron-fed explosion. Here, the leading neutron front is an additional dissipation region maintained in a turbulent state with a strong magnetic field. The n-shell has thickness $\Delta \sim 10^{11}-10^{12} \mathrm{~cm}$, much larger than the thickness of shock fronts, and it may produce a significant synchrotron emission. The magnetic-field problem could be resolved if the afterglow data are fitted by the thin, $\Delta \sim 10^{-4} R$, magneto-active shell with nearly equipartition magnetic field (a similar phenomenological model was recently suggested by Rossi \& Rees 2002). The observed emission can also come from the shocked trail. This mechanism would be similar to the standard model, however, we emphasize important differences: the shock propagates in a relativistically moving, dense, and hot medium - the neutron trail. It might be easier to generate long-lived magnetic fields in such shocks, and this is an issue for a future study.

The neutron impact ceases at $R_{\text {trail }} \approx 10^{17} \mathrm{~cm}$, which can leave an imprint on the observed afterglow. For example, the shock dissipation can have a bump here (Fig. 1), and a spectral transition is also possible. The arrival time of radiation emitted at $R_{\text {trail }}$ is $t_{\text {trail }} \sim\left(1-\beta_{i}\right)\left(R_{\text {trail }} / c\right)$. It may be as long as 30 days or as short as a few seconds, depending on the i-shell velocity $\beta_{i}\left(R_{\text {trail }}\right)$. Remarkably, the new scale $R_{\text {trail }}$ is almost independent of the ambient medium, and its observational signature would give a unique information on the central engine and the Lorentz factor of the explosion. The first early observation of a GRB afterglow (GRB 021004) showed an interesting re-brightening at $10^{3} \mathrm{~s}$. Future systematic studies by Swift will show whether this is a common GRB feature and allow one to test different models. Also, we do not exclude a possible relevance of neutrons to the 20 day bumps observed in a few GRBs, as the time coincides with $R_{\text {trail }} / c$.

Emission signatures of neutrons should be weak or absent if the ejecta are strongly dominated by magnetic fields, with a very low baryon contamination. Then the neutron component decouples early, with a modest Lorentz factor $\Gamma_{n}$, and decays at small radii. $\Gamma_{n}$ can be estimated as $\Gamma_{n} \approx$ $100\left(\dot{M}_{b} / 10^{26} \mathrm{~g} \mathrm{~s}^{-1}\right)^{1 / 3}$, where $\dot{M}_{b}$ is the isotropic equivalent of the baryonic losses of the central engine.

We focused here on the neutron front and did not account for the $\gamma$-ray precursor that impacts the blast wave dynamics at $R<R_{\text {acc }}=0.7 \times 10^{16}\left(E_{\gamma} / 10^{53}\right)^{1 / 2} \mathrm{~cm}$, where $E_{\gamma}$ is the isotropic energy of the GRB. The analysis in this Letter is strictly valid for afterglows emitted at $R>R_{\text {acc }}$. Then the radiation-front effects described in Beloborodov (2002), including the gap opening at $R<0.3 R_{\text {acc }}$, occur at smaller radii, and apply to the earlier afterglow. For a dense medium, where $R_{\mathrm{dec}}<R_{\mathrm{acc}}$, the effects of the neutron and $\gamma$-ray fronts should be studied together. 
I am grateful to P. Goldreich, R. Blandford, and TAPIR group for their hospitality during my

stay at Caltech, where part of this work was done. This research was supported by NSERC and RFBR grant 00-02-16135.

\section{REFERENCES}

Bahcall, J. N., \& Mészáros, P. 2000, Phys. Rev. Lett., 85, 1362

Beloborodov, A. M. 2002, submitted to ApJ, astro-ph/0210522 (Paper 1)

Beloborodov, A. M. 2002, ApJ, 565, 808

Blandford, R. D., \& McKee, C. F. 1976, Phys. Fluids, 19, 1130

Derishev, E. V., Kocharovsky, V. V., Kocharovsky, Vl. V. 1999a, A\&A, 345, L51

Derishev, E. V., Kocharovsky, V. V., Kocharovsky, Vl. V. 1999b, ApJ, 521, 640

Fuller, G. M., Pruet, J., \& Abazajian, K. 2000, Phys. Rev. Lett., 85, 2673

Gruzinov, A. 2001, ApJ, 563, L15

Medvedev, M. V., \& Loeb, A. 1999, ApJ, 526, 697

Mészáros, P. 2002, ARA\&A, 40, 171

Mészáros, P., \& Rees, M. J. 2000, ApJ, 541, L5

Pruet, J., \& Dalal, N. 2002, ApJ, 573, 770

Rossi, E., \& Rees, M. J. 2002, submitted to MNRAS, astro-ph/0204406

Sagdeev, R. Z. 1966, Rev. Plasma Phys., 4, 23 